\title{
L'apprentissage de la culture à travers les techniques du corps : savoir anthropologique chez Claude Simon
}

Cultural Apprenticeship through Bodily Techniques: Anthropological Knowledge in Claude Simon

\section{Cecilia Benaglia}

\section{(2) OpenEdition Journals}

\section{Édition électronique}

URL : http://journals.openedition.org/ccs/2313

DOI : $10.4000 /$ ccs. 2313

ISSN : 2558-782X

\section{Éditeur :}

Presses universitaires de Rennes, Association des lecteurs de Claude Simon

\section{Édition imprimée}

Date de publication : 30 septembre 2019

Pagination : 181-194

ISBN : 978-2-7535-7795-4

ISSN : $1774-9425$

\section{Référence électronique}

Cecilia Benaglia, «L'apprentissage de la culture à travers les techniques du corps : savoir anthropologique chez Claude Simon », Cahiers Claude Simon [En ligne], 14 | 2019, mis en ligne le 30 septembre 2020, consulté le 10 octobre 2020. URL : http://journals.openedition.org/ccs/2313 ; DOI : https://doi.org/10.4000/ccs.2313 


\title{
L'APPRENTISSAGE DE LA CULTURE À TRAVERS LES TECHNIQUES DU CORPS : SAVOIR ANTHROPOLOGIQUE CHEZ CLAUDE SIMON
}

\author{
Cecilia BENAGLIA \\ San Diego State University
}

L'œuvre de Claude Simon se caractérise par une attention presque obsessionnelle aux positions des corps, à leurs mouvements et à la gestuelle des personnages - éléments qui font tous l'objet de longues descriptions dans ses textes. Depuis le début de son parcours d'écrivain, Simon explore les ressources expressives du corps dans leur ampleur, en concevant souvent celui-ci comme un instrument de communication et en traitant le vécu sensoriel et perceptif comme la base sur laquelle fonder l'écriture. Dans ses romans, le langage corporel remplace souvent toute médiation verbale entre les personnages ${ }^{1}$.

La critique a déjà relevé la " densité des références corporelles " (Jean H. Duffy) dans l'œuvre de Simon. Plusieurs travaux ont interrogé, par exemple, la place du corps de l'écrivain dans le processus d'écriture ${ }^{2}$, ou encore les liens existant entre cette ouvre et la pensée de Maurice Merleau-Ponty, qui, dans le contexte de la philosophie occidentale, fait figure de "saint patron " du

1. Dans le cas de la relation adultérine entre Corinne et Iglésia, par exemple, cela donne lieu à la pure rencontre physique entre les amants, qui, dépourvue de toute phase préparatoire, laisse libre cours aux impulsions les plus instinctives. Ainsi, dans La Route des Flandres, le narrateur parle du lien qui existe entre Corinne et le jockey en le décrivant non " pas (comme) [...] une intrigue se déroulant, verbeuse, convenue, ordonnée ", mais plutôt comme de l'amour - ou plutôt la passion -, c'est-à-dire " cette chose muette, ces élans, ces répulsions, ces haines, tout informulé - et même informé -, et donc cette simple suite de gestes [...], et, au centre, sans préambule, cet assaut, ce corps à corps » $(R F$, p. 225-226).

2. P. Dirkx, "Claude Simon : antinomie et corps écrivant ", dans Claude Simon : Situations, Lyon, ENS éditions, 2012, p. 179-197. 
corps $^{3}$. Jean H. Duffy, portant son regard plus précisément sur les romans des années 1970, a analysé la place des descriptions du corps lorsque celui-ci arrête de fonctionner correctement, à cause, notamment, de la maladie, qui, de médium invisible, le transforme en obstacle opaque concentrant sur lui la conscience du sujet souffrant ${ }^{4}$. La réflexion simonienne sur la corporéité passe en effet par la reconnaissance de la faiblesse du corps, ce qui rapproche ultérieurement son œuvre de la pensée de Merleau-Ponty qui considère même les défaillances corporelles comme des éléments positifs, en ce qu'elles confèrent au corps sa capacité particulière de dépeindre fidèlement " un monde d'expérience lui-même ambigu, vague et perpétuellement changeant ${ }^{5}$ ".

Dans les pages qui suivent, nous voudrions étudier le lien entre corps et connaissance tel qu'il est exploré dans l'œuvre de Simon, afin de prolonger l'analyse du rôle de la corporalité comme véhicule d'une pensée sur le monde et sur soi. Dans les romans simoniens, en plus d'être un vecteur de communication entre les personnages, le corps devient en effet un véritable instrument de connaissance et de compréhension du réel. Ainsi, malgré la distance qui les sépare, Montès et Hélène, dans Le Vent, ont en commun la capacité d'approcher le monde par les sens, sans la médiation de l'intelligence. Pour Hélène, il s'agit là d'une qualité qui découle de sa nature féminine, qui lui permet de " classer » les gens « du premier coup d'œil », ou bien de deviner les plans de sa sœur Cécile sans besoin de la suivre, ni de lui poser des questions, "se contentant le soir de cet examen fulgurant (pas l'intelligence, la déduction, même pas l'observation : quelque chose de plus profond, quelque chose du corps, de la chair $[\ldots]) »(V$, p. 169). De Montès, le narrateur souligne l'« inaptitude fondamentale à prendre conscience de la vie, des choses, des événements, autrement que par l'intermédiaire des sens, du cœur»( $V$, p. 114). Et enfin, dans L'Herbe, Louise voit « non par l'intermédiaire de ses yeux mais au moyen de cette connaissance du corps qui peut assister comme de l'extérieur à ses propres mouvements " (Herbe, p. 131 - nous soulignons).

3. L'expression est de Richard Shusterman, dans "Silence du corps, claudication du philosophe : le déficit d'attention somatique chez Merleau-Ponty ", Conscience du corps. Pour une soma-esthétique, trad. N. Vieillescazes, Éditions de l'éclat, 2007, p. 73-107. Sur les liens entre l'œuvre simonienne et la phénoménologie de Merleau-Ponty voir J. H. Duffy "Claude Simon, Merleau-Ponty and perception ", dans French Studies: A Quartely Review, 46, janvier 1992, p. 33-52.

4. J. H. Duffy, "Corporéité, métaphore et image dans Orion aveugle et Les Corps conducteurs de Claude Simon ", Cahiers Claude Simon, n 9, 2014, p. 117-141. Voir aussi Cahiers Claude Simon, n 12 ("Corps et matière »), 2017.

5. R. Shusterman, Conscience du corps, éd. cit., p. 77. 
Montès, Hélène et Louise incarnent un type de rapport au monde fondé sur ce que Pierre Bourdieu, avec une formule qu'on vient de trouver à un mot près chez Simon, appelle une " connaissance par corps ${ }^{6} »$. À savoir une connaissance pratique qui, n'accédant pas au monde par la médiation de la raison, nous fait appréhender la structure et l'image de celui-ci à travers le filtre ou le prisme des sens. Ce type de connaissance est souvent le seul à disposition des personnages simoniens. Ceux-ci sont maintes fois confrontés à des situations traumatisantes ou de dysfonctionnement physique ayant comme conséquence de mettre hors d'usage le raisonnement, et de transformer par là le corps en instrument de compréhension privilégié. Tel Montès, les personnages simoniens regardent le monde "sans que rien d'autre (aucun concept, aucune idée, à plus forte raison aucune pensée) ne s'ensuivît dans [leur] esprit : rien d'autre que la simple conscience des formes, des objets» $(V$, p. 151).

Notre lecture s'inscrit dans un examen plus large portant sur les manières dont l'œuvre simonienne véhicule une vision du monde fondée sur un savoir de nature pratique plutôt que discursive, associé à un "faire " plutôt qu'à un "dire ". Autrement dit, un savoir-faire enfoui dans les corps et souvent inconscient, se démarquant de la conception prépondérante du savoir entendu comme ensemble organisé de connaissances appréhendées par l'esprit. Merleau-Ponty situait la " profonde nouveauté » de l'œuvre simonienne dans sa capacité à dire le contact du sujet avec les choses et à "s'adresse(r) à cette région au-dessous des idées " ", hors des pistes de l'explicitation. Mais cette ouvre, par son travail d'enregistrement et de prise en compte des mouvements inconsciemment appréhendés et plus largement des états préréflexifs, est porteuse également de ce que Richard Shusterman appellerait une " réflexion somatique ${ }^{8}$ " permettant une prise de conscience corporelle réflexive. Tout en restant la plupart du temps en deçà de l'interprétation, l'écriture littéraire rend sensible la manière dont les événements affectent les corps et la perception des personnages, et contribue par là à l'élucidation des mécanismes psychophysiques et des liens entre cognition et corporéité.

6. Dans P. Bourdieu, Méditations pascaliennes, Paris, Le Seuil, 1997. Voir en particulier p. 185-229.

7. M. Merleau-Ponty, Notes de cours «Sur Claude Simon », présentation par S. Ménasé et J. Neefs, Genesis, n 6,1994 , p. 136.

8. Shusterman parle du soma à partir de la définition du même terme fournie par Thomas Hanna : «Hanna définit le soma comme "un corps perçu de l'intérieur" ("the body experienced from within"). Je comprends le soma comme corps vivant, sentant, avec une intentionnalité qui implique l'activité " (B. Andrieu, "Entretien avec Richard Shusterman ", Corps, no 6, 2009/1, p. 5-10). Voir aussi Conscience du corps, éd. cit., en particulier le chap. 2, consacré à Merleau-Ponty, p. 73-107. 


\section{TECHNIQUES DU CORPS ET SAVOIR INCORPORÉ}

Afin de mettre en lumière cette forme de savoir recelée dans l'œuvre, nous fonderons notre analyse sur la notion de technique $d u$ corps, élaborée par l'anthropologue et sociologue Marcel Mauss, dont les travaux constituent d'ailleurs des références fondamentales pour les réflexions de Bourdieu et de Shusterman. Mauss, qui présente cette notion lors d'une conférence prononcée en 1934, entend par techniques du corps "les façons dont les hommes, société par société, d'une façon traditionnelle, savent se servir de leur corps ${ }^{9}$ ". Il y a technique, bien sûr, en présence d'instruments, mais les outils ne sont pas nécessaires pour qu'on puisse parler de technique, puisque Mauss considère le corps lui-même comme un instrument - le premier et « le plus naturel » dont dispose l'homme. C'est d'ailleurs dans cet élargissement au corps de la notion de technique que se trouve la contribution majeure du travail de l'anthropologue, pour qui, dès lors, il y a technique chaque fois qu'il y a un rythme et un ordre dans les mouvements corporels, chaque fois qu'un dessin particulier est exécuté par ces mouvements en vue d'un objectif précis. Parmi les techniques que Mauss prend en considération, et dont il analyse l'évolution et les changements d'une société à l'autre, se trouvent la nage, la marche, le saut, la danse, les manières dont on mange et dont on dort, et bien d'autres actions quotidiennes. Bref, Mauss entend cette notion au sens large, comme l'emploi intelligent et ordonné du corps. On peut comprendre une technique corporelle aussi comme le résultat d'une "incorporation ", à savoir d'un " processus d'intégration à un corps humain d'objets ou de symboles ${ }^{10}$ " ayant comme objet des gestes et des mouvements. Ce véritable savoir inscrit dans l'individu a une importance cruciale, car, loin d'être simplement utilitaire pour l'homme, il finit par modifier la nature même de ce dernier dans la mesure où il existe " entre l'homme et les techniques une causalité réciproque : il les fait et elles lui permettent de se faire ${ }^{11}$ ".

En reconstruisant le parcours qui l'a mené à l'élaboration de cette notion, Mauss rappelle à quel point ce lent travail de conceptualisation s'est fait à

9. M. Mauss, Techniques, technologies et civilisation, PUF, 2012, p. 366. Le texte de la conférence a été publié pour la première fois dans le Journal de Psychologie, XXXII, nº 3-4, 1936.

10. P. Dirkx, entrée "Incorporation », dans A. Glinoer et D. Saint-Amand (dir.), Le Lexique "Socius », [http://ressources-socius.info/index.php/lexique/21-lexique/187-incorporation], page consultée le 29 juillet 2018.

11. Mauss cité par N. Schlanger dans «Une technologie engagée. Marcel Mauss et l'étude des techniques dans les sciences sociales " (M. Mauss, Techniques, technologies et civilisation, PUF, 2012, p. 73). 
partir de l'observation d'actes et de faits épars, constituant des aspects difficilement repérables, car non spectaculaires, de la vie quotidienne des hommes en société. Son travail a consisté en grande partie à isoler et réévaluer un ensemble de phénomènes qui étaient traditionnellement regroupés, par les sciences sociales, sous la "vilaine rubrique " de " Divers ». Or, tout ce qui est classé dans cette catégorie de « divers " pourrait être considéré comme faisant partie également du territoire exploré par la littérature, qui, souvent, se risque dans l'observation des comportements et des activités humains les moins étudiés ${ }^{12}$. C'est ainsi en référence à Mauss et aux techniques corporelles que Georges Perec évoquait des domaines laissés encore en friche à partir desquels on peut écrire une histoire du corps. Perec définissait ces espaces comme des

zones d'urgence dont on sait seulement qu'on ne sait pas grand-chose, mais dont on pressent qu'on pourrait beaucoup y trouver si l'on s'avisait d'y prêter quelque attention : faits banals, passés sous silence, non pris en charge, allant d'eux-mêmes, ils nous décrivent pourtant $[\ldots]$ ils renvoient à l'histoire de notre corps, à la culture qui a modelé nos gestes et nos postures, à l'éducation qui a façonné nos actes moteurs au moins autant que nos actes mentaux ${ }^{13}$.

S'inscrivant dans une perspective semblable, l'œuvre de Simon accorde une attention considérable à ces "zones d'urgence ", zones constituées par le côté muet de l'existence, par tout ce que la parole ne saurait prendre en charge et exprimer. Son ouvre explore la place des corps au sein de cet espace relationnel qu'est la société et, à travers une logique indiciaire cumulant les détails composant l'existence ordinaire, interroge les manières dont le passé s'inscrit dans les individus et dont la culture est " apprise " à travers des mécanismes souvent automatisés, agissant hors de toute prise de conscience ${ }^{14}$. Ainsi la précision qui, dans ses romans, caractérise la description des positions, des dimensions et des formes des corps, de même que l'enregistrement de leurs mouvements dans l'espace, est nécessaire à l'économie narrative. Non pas pour reconstruire un scénario ni pour poser un décor : cette précision est plutôt dictée par la conviction que c'est dans les dispositions spatiales et les conduites corporelles que se tient l'accès à la connaissance d'un individu, et

12. Voir Carlo Ginzburg qui parle du paradigme indiciaire de l'observation littéraire dans « Traces. Racines d'un paradigme indiciaire " (C. Ginzburg, Mythes, emblèmes, traces; morphologie et histoire, Grasse, Verdier, 2010). Sur la portée sociologique de l'esthétique, voir aussi M. Macé, Façons de lire, manières d'être, Gallimard, 2011 et B. Carnevali, "Mimesis littéraire et connaissance morale. La tradition de l'"éthopée" ", dans Annales. Histoire, Sciences sociales, 2010/2, p. 291-322.

13. G. Perec, Penser/Classer, Le Seuil, 1985, p. 109.

14. Voir à ce propos W. Nitsch, "Une poétique de la dépense. Claude Simon et le Collège de Sociologie », Cahiers Claude Simon, no 4, 2008, p. 33-52. 
que toute condition morale et existentielle est inséparablement une condition physique, et vice-versa.

\section{TECHNIQUES DU CORPS,}

\section{CONTEXTE GUERRIER ET DISCIPLINE MILITAIRE}

Dans le texte de la conférence de 1936, Mauss remarque à quel point sa participation à la Première Guerre mondiale a été fondamentale pour avancer dans la compréhension de la notion de technique du corps. Son expérience au front et dans l'armée lui a fourni, en effet, un réservoir important d'exemples et a été l'occasion d'observer l'homme dans des situations exceptionnelles où le corps était la seule vraie ressource pour la survie. Les conditions extrêmes du combat des tranchées lui ont appris personnellement, par exemple, des techniques du sommeil insolites ("Je peux vous dire que la guerre m'a appris à dormir partout, sur des tas de cailloux par exemple »), le convainquant du fait que « la notion que le coucher est quelque chose de naturel est complètement inexacte » et que, plus généralement, il n'existe peut-être pas de "façon naturelle " chez l'adulte ${ }^{15}$. On sait que Simon accordait lui aussi à la guerre le statut d'observatoire privilégié du comportement humain, à la fois moral et physique : la guerre est " le seul endroit où l'on puisse bien voir certaines choses ", écrivait-il dans La Corde raide en 1947 (p. 54). La guerre lui apparaissait comme un formidable observatoire des comportements humains et, de ce fait, elle est omniprésente dans ses romans, à la fois comme thématique et comme contexte. Et il faut parler de guerres, au pluriel, car l'écrivain se penche tout aussi bien sur les conflits auxquels il a participé personnellement que sur ceux qu'il a vécus par l'intermédiaire de l'histoire familiale. Les personnages qui sont confrontés à la guerre vivent des situations qui les plongent dans un état psychophysique que le narrateur des Géorgiques évoque à travers l'expérience de $\mathrm{O}$. et de ses deux compagnons. Ceux-ci développent des "réflexes [...] de vigilance, d'instinctive alarme ", et leurs chairs et muscles ne sont plus commandés

par un cerveau en proie au désarroi ou au désespoir (ils n’en avaient pas ou plutôt plus le temps) mais uniquement occupé des moyens de survivre, repoussant à plus tard tout questionnement $[\ldots]$ ne faisant plus confiance maintenant quà leurs sens tenus constamment en éveil. $(G$, p. 815$)$ 
Cette attention et cette disponibilité extrêmes du corps en situation d'alerte mènent ce dernier à adopter spontanément ce que, en empruntant la notion de Mauss, on peut appeler de véritables techniques. Ces dernières servent pour survivre, pour combattre, et pour accomplir quelques-unes des actions que $\mathrm{S}$. évoque dans Le Jardin des Plantes en présence du journaliste : "de courir, ou de galoper, de se jeter par terre, de se relever, de remonter à cheval, de sauter de nouveau à terre, de courir à quatre pattes " (p. 958). Ces mouvements et ces activités, apparemment si ordinaires et triviaux, sont en réalité le résultat d'un long travail d'apprentissage qui impose au corps, en l'occurrence au corps masculin, un ordre capable de limiter toute réaction instinctive, et d'augmenter ses capacités d'endurance. C'est sans doute suite à un considérable travail de dressage corporel que les deux personnages des Géorgiques peuvent faire preuve d'une extrême adaptabilité leur permettant de dormir n'importe où, dans une série d'endroits dont le narrateur livre la liste détaillée. D’abord en parlant du général L.S.M., à propos duquel on lit : "Il couche dans des palais. Il couche dans des étables. Il couche dans les bois. Il couche sous la tente "; puis, en italiques, se référant à l'autre personnage, le volontaire de la guerre d'Espagne: "Il couche dans une église incendiée. Il couche dans un terrain vague, [...] dans un chantier abandonné, recroquevillé dans l'escalier d'un abri antiaérien [...] Il couche à même le sol» (G, p. 661).

Une autre sphère d'activités liées à la guerre et dont la description occupe une place centrale dans l'œuvre simonienne est celle de la discipline militaire. L'attention portée à ce domaine découle de l'histoire familiale même de l'écrivain, marquée par une participation à l'armée s'étalant sur plusieurs générations et par " le culte des valeurs militaires ${ }^{16}$ ". Ainsi, tout particulièrement dans La Route des Flandres, dans Les Géorgiques et dans Le Jardin des Plantes, sont innombrables les descriptions minutieuses d'opérations, qui vont des plus banales (desseller un cheval) aux plus complexes (installer un bivouac, diriger une manœuvre d'artillerie), qui remplissent de nombreuses journées, et qui occupent les simples soldats tout autant que les plus hauts gradés ${ }^{17}$. Car, si le savoir militaire peut être celui, conceptuel, des cartes et des plans stratégiques décrits dans Les Géorgiques, il inclut également le dressage corporel imposé aux combattants à travers une discipline fondée sur des codes et

16. V. Berne, "La bataille de la phrase" : archives militaires et langage romanesque dans l'œuvre de Claude Simon ", Revue historique des armées, n 277, 2014/4, p. 105.

17. Voir, par exemple, la description de la mise en place des tentes dans Le Jardin des Plantes, p. 10801081, ou dans Les Géorgiques, p. 888, la description d'un régiment d'artillerie. 
des règles très rigidement établis. Une discipline qui a comme but de former des corps programmés pour le combat et la survie. Ainsi, dans Le Jardin des Plantes, les recrues du régiment s'exercent au " maniement d'armes " consistant en des exercices dont " le plus redouté " est "l'école de sabre où, en formation espacée sur le champ de manœuvre, les cavaliers répétaient jusqu’à épuisement coup de pointe en avant vers la droite, coup de pointe en avant vers la gauche, coup de pointe sur un ennemi à terre " $(J P$, p. 1045). La description que Simon livre des soldats dans La Route des Flandres saisit l'un des aspects les plus importants caractérisant ce type de dressage, et donne à voir ses effets durables. Les soldats accomplissent "spontanément et sous leur propre autorité et contrôle la suite des mouvements auxquels leurs cerveaux ne semblaient pas prêter attention " $(R F, \mathrm{p} .367)$. Les soldats n'ont plus besoin de penser aux mouvements, car leur corps pense à la place du cerveau, ayant déjà intériorisé, suite à un long entrainement, les gestes à accomplir. D'une manière similaire, l'ordre et un certain maintien correspondant à son rang sont inscrits une fois pour toutes dans le corps du capitaine de Reixach, qui, malgré la défaite imminente et après l'anéantissement de son escadron, ne renonce pas à "se tenir toujours droit et raide sur sa selle aussi droit et aussi raide que s'il avait été en train de défiler à la revue du 14 juillet " $(R F$, p. 202). Le Palace offre un autre exemple d'un personnage se trouvant dans une situation marquée par une tension comparable à celle du combat, à savoir le moment de l'assassinat du chef fasciste italien par l'anarchiste nommé "l'homme-fusil ». Le narrateur relate cet épisode en insistant sur la dissociation entre, d'une part, le corps du personnage, habité par le traumatisme de l'acte violent qu'il est sur le point d'accomplir, et, d'autre part, sa conscience et sa volonté. Le meurtre commis, "son corps (ses jambes, son cour, ses poumons) continua[it] à courir pour ainsi dire en dehors de lui [...] pendant que son esprit parfaitement calme, immobile, était en train de prendre conscience de cela » $(P$, p. 469). Le contrôle que l'homme-fusil peut exercer sur ses mouvements est constamment mis en doute : " ce qui signifiait qu'il avait eu le temps, lui, de faire cela : commander à ses muscles, et les muscles celui d'obéir, d'exécuter - à moins que les jambes se fussent tendues toutes seules, se fussent chargées sous leur propre responsabilité de le soulever » $(P$, p. 455). D'une manière similaire, ce n'est pas lui qui fait le geste de tirer, mais bien "sa main toute seule, [...] en train de faire donc ce qu'il croyait ne pouvoir jamais arriver à faire, ce que sa main et son œil exécutaient mais pas lui..." $(P$, p. 456). Tous ces exemples suggérant la séparation entre le 
sujet - « lui » - et son corps - «sa main et son œil » - indiquent que le corps, agissant en dehors de la conscience éveillée, sait et fait quelque chose de plus et de différent dont l'esprit, se trouvant dans une position subordonnée, n'a qu'à prendre acte, étonné de ce qui vient d'avoir lieu.

\section{SENS PRATIQUE ET SURVIE : GASTONE NOVELLI AU BRÉSIL}

Le sens pratique que les personnages simoniens déploient, acquis grâce à l'entrainement, l'observation et l'imitation et "passé au stade de réflexe ", se trouve dans l'œuvre en opposition avec un savoir qu'on peut définir, au contraire, comme intellectuel et abstrait. Cette opposition est de différentes manières thématisée et explicitée dans les romans, où le narrateur et les personnages penchent souvent pour ce savoir "pratique ", acquis par mimétisme, et critiquent, voire ridiculisent, le savoir intellectuel, acquis par la lecture et l'étude. Que l'on pense à l'opinion que Georges exprime sur Pierre, ou à Blum dont le narrateur nous dit qu’il " possédait héréditairement une connaissance (l'intelligence avait dit Georges, mais ce n'était pas seulement cela : plus encore : l'expérience intime, atavique, passée au stade du réflexe, de la stupidité et de la méchanceté humaines) des choses qui valait bien trois fois celle qu'un jeune homme de bonne famille avait pu retirer de l'étude des auteurs classiques français, latins et grecs " $(R F$, p. 309). L'axiologie qui soustend ce passage et qui pose la supériorité de la connaissance issue du corps sur le savoir intellectuel va de pair avec le rapport ambivalent que Simon entretient avec la tradition humaniste et la critique qu'il avance de la posture intellectuelle. Ces positions ont des racines profondes dans l'œuvre et on les rencontre dès les premiers romans, comme Le Sacre du printemps, où Simon imagine " un type qui aurait appris le ski, ou la natation, dans une de ces brochures spécialisées et qui se trouverait tout à coup, avec ses connaissances théoriques et livresques, tout meurtri sur la neige, suffocant dans l'eau " (SP, p. 160). Ce type se retrouverait finalement à "donn[er] tort aux livres et à ceux qui les avaient écrits ", car les livres, et le savoir qu'ils contiennent, représentent en fin de compte une trahison : ils ne tiennent pas leurs promesses et laissent le sujet démuni et profondément dépourvu face au monde. La critique de la posture intellectuelle est ancrée, ensuite, dans la conviction profonde chez Simon qu'il existe un lien indéniable entre le système de connaissance sur lequel se fonde la civilisation européenne et les catastrophes 
des Première et Deuxième Guerres mondiales ${ }^{18}$. Les notions de culture, de civilisation et d'humanisme, devenues suspectes, font l'objet d'une mise à distance sinon d'un refus, qui mène l'écrivain à réévaluer ce savoir-faire " autre ", renvoyant à un état primitif de la vie sur terre, mais qui est jugé comme tout aussi ordonné, rigoureux et efficace que le savoir théorique.

Dans Le Jardin des Plantes, un personnage en particulier incarne cette position : le peintre italien Gastone Novelli. Après la guerre et une période de captivité passée dans un camp de concentration nazi, Novelli fuit en Amérique du Sud afin de rompre tout contact avec la société occidentale et l'ensemble de ses connaissances, qu'il refuse en bloc. Simon relate en particulier un épisode qui a lieu au cours d'une expédition dans la forêt vierge brésilienne. Le peintre est obligé de faire halte pendant plusieurs semaines pour secourir son compagnon qui est malade et ne peut pas voyager. Ayant perçu la présence des habitants de la forêt et ayant compris qu'il était en train d'occuper leur territoire, Novelli se voit contraint d'élaborer petit à petit de véritables techniques de survie. Ce qui le sauve est sa capacité à déchiffrer le comportement non verbal des indigènes, véhiculé par leurs gestes, et à produire en retour des mouvements ou des actions, qui manifestent aux membres de la tribu ses intentions pacifiques. Pendant plusieurs longues journées, Novelli, par une extrême maîtrise de soi, s'abstient « soigneusement de laisser paraître, soit par son attitude, soit par des gestes ou des regards, le moindre sentiment de surprise ou de crainte" ( $J P$, p. 1075). Il survit finalement grâce à la répétition étudiée d'une série d'actes qui expriment une attitude composant un parfait équilibre entre la faiblesse et la force excessive. Car, comme l'explique le narrateur, il se souvenait avoir appris à Dachau que "si tu as l'air trop fort, on te tue parce qu'on a peur de toi; [...] si tu as l'air trop faible, on te tue aussi, cette fois simplement pour s'amuser" (JP, p. 1074). La situation type dans laquelle se trouve Novelli, caractérisée par l'impossibilité complète de toute communication verbale, met en scène une forme d'apprentissage opposée à celui qu'on peut faire assis à une table d'écolier. Novelli apprend à survivre sur le terrain, et dans ce processus, il peut compter exclusivement sur les ressources du corps pour construire une forme de communication basée sur une série de mouvements ordonnés et répétés - formant en somme un rythme qui devient un langage.

18. Voir à ce propos C. Yapaudjian-Labat, "L'humanisme en question ", dans Claude Simon : Situations, éd. cit., p. 103-116. 


\section{L'ART ÉQUESTRE ET LA TRANSMISSION INÉGALE DES TECHNIQUES}

Selon une définition plus succincte que Mauss fournit des techniques, ces dernières sont des " actes traditionnels efficaces", et en vertu de leur efficacité font l'objet, comme tout savoir utile, d'un enseignement, d'un apprentissage et d'une transmission : "L'enfant, l'adulte, imite des actes qu'il a vu réussir par des personnes en qui il a confiance et qui ont autorité sur lui. L'acte s'impose du dehors, d'en haut, fût-il un acte exclusivement biologique, concernant son $\operatorname{corps}^{19}$. " Si le corps est le premier et le plus naturel instrument de l'homme, il existe donc un art d'utiliser cet instrument, art né de l'accumulation du savoir pratique au fil des générations et devenant dès lors partie intégrante du processus d'éducation et d'acquisition d'une culture donnée. Dans l'œuvre simonienne, on trouve un bon exemple de techniques conçues comme un art dans le domaine de l'équitation. L'attention que Simon porte à ce qu'il appelle justement l'" art équestre " découle d'une familiarité qui remonte à l'adolescence de l'écrivain - "J'ai été élevé par un ancien officier de cavalerie On m'a mis sur un cheval à douze ans " (JP, p. 978) - et qui est prolongée à l'âge adulte par son expérience en tant que cavalier dans les dragons. Les pages de ses romans sont empreintes d'une sensibilité et d'une affection pour les chevaux, qui font par ailleurs partie de la « composante érotique " de l'imaginaire littéraire de l'écrivain ${ }^{20}$. Lélégance et l'habileté des cavaliers, qui font corps avec l'animal qu'ils chevauchent, suscitent aussi l'admiration. Dans Le Jardin des Plantes, Simon cite le général L'Hotte, " [l'] un des penseurs les plus réputés de l'équitation française ", pour qui « les trois objectifs du cavalier doivent être d'obtenir un cheval "calme, en avant, droit” " (JP, p. 951). Quelques lignes plus loin, il donne l'exemple de deux officiers dont " les hanches [...] absorbent si parfaitement les mouvements de leurs montures que leurs dos restent absolument droits, rigides, comme si les deux hommes se déplaçaient assis sur un véhicule monté sur rails » ( $J P$, p. 951). Combien de savoir recèle un mouvement des bras, des hanches ou du cou? Les descriptions de Simon permettent de prendre la mesure de cette dimension, souvent invisible à l'œil du profane, et montrent des cavaliers qui, étant " au sommet de [leur] art : marcher sans faire une faute" (JP, p. 952), font de cette maîtrise un objet d'orgueil et de mise en scène.

19. M. Mauss, op. cit. p. 372.

20. À propos de la place des animaux dans l'œuvre, voir M. Miguet-Ollagnier, «Animaux : les autres moi de Claude Simon ", dans L'Animal littéraire. Des animaux et des mots, Éditions universitaires de Dijon, 2010, p. 154. 
L'art d'employer son corps étant transmissible de génération en génération, il ancre l'homme dans un passé plus ou moins ancien, et transforme le corps en un réservoir de mémoire, toujours inséparablement individuelle et collective. Les personnages des romans simoniens sont tous ou presque porteurs d'un passé, souvent évoqué et exprimé à travers des gestes ou des postures qu'ils ont repris à des devanciers et qui seront repris par des successeurs. De Reixach, dans La Route des Flandres, est peut-être le personnage qui illustre au mieux ce qu'on pourrait appeler une stratification des temporalités historiques faite corps. Il se trouve au beau milieu de la route et, face aux balles allemandes, il brandit son sabre. Ce " geste héréditaire de statue équestre ", véritable technique " que lui avaient probablement transmis[e] des générations de sabreurs" $(R F$, p. 199) n'a aucune fonction protectrice. De Reixach l'accomplit uniquement afin de convoquer l'ancêtre, d'instaurer une répétition qui inscrit le moment présent dans l'histoire de la famille. Ces renvois à d'autres temporalités, très fréquents dans l'œuvre, peuvent aussi être suscités par des personnages marginaux, comme, par exemple, une vieille femme que Georges rencontre et dont la voix est comparée à celle des « pleureuses de l'Antiquité " accomplissant leurs chants rituels, image qui rattache le moment présent à une histoire ancienne et plonge le récit dans une temporalité presque mythique.

Bien que les techniques constituent le patrimoine partagé par une société dans son ensemble, certaines d'entre elles se transmettent à l'intérieur d'espaces sociaux exclusifs. C'est le cas, justement, des techniques équestres, dont la maîtrise, qui se lègue de génération en génération à l'intérieur d'un même groupe restreint ou d'une même famille, marque l'appartenance à un clan prestigieux, à une élite. Ce qui nous conduit à un dernier temps de notre réflexion, qui concerne les manières dont la corporalité et la maitrise du corps sont à la fois le résultat et l'expression d'une appartenance sociale. Nos actions quotidiennes les plus " naturelles " laissent des signes visibles sur les corps, dans la tenue, dans l'attitude, dans la conduite, qui deviennent avec le temps des "stigmates" $(G$, p. 694) dessinant l'identité sociale de tout individu. Que l'on pense aux tantes Marie et Eugénie dans L'Herbe, dont les robes, et surtout la manière dont elles sont confectionnées, signalent l'origine sociale modeste. Autre exemple tiré des Géorgiques: le narrateur évoque les soldats qui partent au front comme appartenant à deux groupes distincts. On trouve, d'une part, une « sorte de société secrète [...] entre ceux d'origine paysanne : leur même comportement à l'égard des chevaux, à la fois respectueux (valeur 
marchande) et empreint d'une manière de tendresse, de complicité, de compagnonnage héréditaire pour ainsi dire. Même chose (même ancestrale familiarité) à l'égard des outils (fourches, brouettes), des litières, du fumier, du foin, de l'avoine " $(G$, p. 694). On trouve d'autre part les soldats « des villes " qui sont "dépaysés dans cet univers anachronique de bêtes et d'armes, se protégeant, se fabriquant une puérile cuirasse de grossièreté canaille qui se traduit par un vocabulaire argotique à l'excès " (ibid.). La distance entre ces deux groupes est exprimée exclusivement à travers les effets que le rapport (voire l'absence de rapport) entretenu avec les instruments de travail, les armes et les animaux, a sur leur comportement.

\section{LE NON-SAVOIR DU CORPS}

La réflexion de Mauss sur les techniques du corps défiait très consciemment la réticence que la société occidentale contemporaine montrait, en général, envers les occupations manuelles, matérielles, considérées comme basses et " non-intellectuelles ${ }^{21}$ ». L'œuvre de Simon, fondée sur une «éthique de la matérialité 22 ", creuse la voie ouverte et parcourue par l'anthropologue, en réévaluant l'Homo faber au détriment de l'Homo sapiens. Dans "La fiction motà-mot ", Simon considérait le roman moderne comme le lieu d'un non-savoir, sans s'exprimer plus avant sur la signification de ce terme ${ }^{23}$. On peut faire correspondre à ce non-savoir précisément cet autre savoir du corps, qui se montre dans l'œuvre à travers des éléments dispersés et fragmentaires, que la notion de techniques corporelles, c'est du moins notre hypothèse, aide à lire d'une manière plus cohérente. Si pour la tradition philosophique occidentale, ce type de savoir est illégitime et considéré négativement, pour Simon, comme pour Mauss, cette hiérarchie de valeurs est renversée. Dans tous les exemples que nous avons pris en considération, le corps devient l'ultime réservoir de résilience, il est ce qui reste et résiste quand tout autour s'écroule. Parmi les "valeurs sûres " $(R F$, p. 348) auxquelles il faut retourner après l'effondrement de la société occidentale, il faut inclure à coup sûr les ressources corporelles, le corps pensant.

21. Voir à ce propos N. Schlanger, "Introduction ", dans M. Mauss, Techniques, technologie et civilisation, éd. cit., p. 1-10.

22. Voir D. Viart, Une mémoire inquiète. La Route des Flandres de Claude Simon, PUF, 1997.

23. Dans « La fiction mot à mot ", Simon cite Olga Bernal qui écrit : "Si le roman du XIXe siècle était un roman du savoir, le roman moderne est essentiellement un roman du non-savoir » (EI, p. 1192). 
Simon nous dit, plus généralement, que les phénomènes matériels et physiques méritent la même attention que les phénomènes de la pensée et surpassent même ces derniers en importance. Son œuvre tout entière semble résonner avec ces mots du narrateur de La Route des Flandres, à propos de Georges qui se trouve avec beaucoup d'autres dans un wagon de prisonniers : "il n'était pas dans ses intentions de philosopher ni de se fatiguer à essayer de penser à ce que la pensée était incapable d'atteindre ou d'apprendre, car le problème consistait plus simplement à essayer de dégager sa jambe » $(R F$, p. 243). 